\title{
PERFORMANCE ANALYSIS OF FRACTIONAL ORDER CIRCUITS USING FRACTIONAL ORDER DEVICES (FODs)
}

\author{
Madhab Chandra Tripathy \\ Asst. Professor, College of Engineering and technology (CET) \\ Bhubaneswar, Odisha, India
}

\begin{abstract}
The design and realization of continuous- time fractional order circuits and their performance analysis have been presented. Here, a fractional order devices which is primarily used for realization of fractional order circuits, are described. Also a comparison between the fractional order circuits and its integer order counterpart has been presented. It can be shown by simulation that the fractional order circuits can be used for high frequency application without changing $R C$ time constant of the circuits .This helps to design a high speed analog circuits as compared to classical integer order circuits.
\end{abstract}

Keywords: Fractional order devices (FODs), fractance, constant phase element(CPE) fractional order circuits (FOC), Poly Methyl Methecrylate ( PMMA), sallen-key (KRC) filter, cut-off and Peak Frequency

Cite this Article: Madhab Chandra Tripathy, Performance Analysis of Fractional Order Circuits Using Fractional Order Devices (FODs), International Journal of Electrical Engineering \& Technology, 10(3), 2019, pp. 36-44.

http://iaeme.com/Home/issue/IJEET?Volume=10\&Issue=3

\section{INTRODUCTION}

Now a days, fractional order circuits (FOC) and its design are emerging areas in signal processing engineering. Whether passive or active, the resistors and capacitors are the key components to design Fractional order circuits, Traditional analog FOCs are of integer order, since their transfer functions contain integer powers of $s$. With the advent of fractional order devices (FODs), there has been a growing interest among researchers to examine its effect in fractional order circuits.. In recent time a PMMA coated Constant Phase devices ( FOD) [1, 9] which has been presented in the following section that gives performance fairly close to the behaviors of an ideal fractional order device or fractance in a particular frequency bandwidth. This presented FOD $[1,9]$ is based on metal-insulator-liquid interface and was used to realize a fractional order circuit [1]. The impedance behavior of this shows the phase angle remaining constant over a broad range of frequencies between 0 and $90^{\circ}$.

The mathematical expression for the impedance $(\mathrm{Z})$ of a FOD can be represented as $\mathrm{Z}=\mathrm{Qs}^{-}$ ${ }^{\alpha}$, where $\mathrm{Q}$ is the fractional admittance of the FOD and $\alpha(0<\alpha \leq 1)$ is its order. In such 
element the phase difference between the voltage across its two terminals and current entering these terminals is $-\pi \alpha / 2$. The special cases $\alpha=0,-1,1$ corresponds to the classical resistor, inductor and capacitor [1].

Recently, it has been reported in [4] about design and simulation of fractional order circuit using a single fractional order devices and then simulation of $2^{\text {nd }}$ order circuit using two fractance $[2,6]$ both having exponent $(\alpha)$ and impedance $(Z)$. In this report, we explore to generalize classical integer order circuits to fractional domain because it is less sensitive towards passive component variations [12]. Here it can be shown that the fractional order circuits can be used for high frequency by reducing the order $(\alpha)$ of FOD, but not through reducing the time constant $R C$ of the circuit. Also these circuits have higher gain stability as compared to conventional integer order circuit. Due to the above facts, the fractional order circuits are becoming more popular.

This paper is organized as follows: Sect.2 presents the background of fractional order devices. Sect. 3 presents the comparison between the fractional order circuits and its integer order counterpart through simulation results has been presented. Concluding remarks are summarized in Sect.4.

\section{BACK GROUND OF FRACTIONAL ORDER DEVICES (FODs)}

Here a fractional order device which is basically used to realized FOC, is described that exhibits constant phase element (CPE) behavior with a polymer coating.

The FOD shown in fig. 1 is made of about $6 \mathrm{~mm}$ wide strip cut out from about a $1.62 \mathrm{~mm}$ thick plate generally that is made used as double sided printed circuit board. The copper cladding on the external faces serves as the electrodes of the FOD. Insulation is provided in order to protect the metal electrode from the environment. This whole thing was taken as substrate. Over this substrate, coating of polymer was made. Here the coating is done with Poly Methyl Methecrylate or .

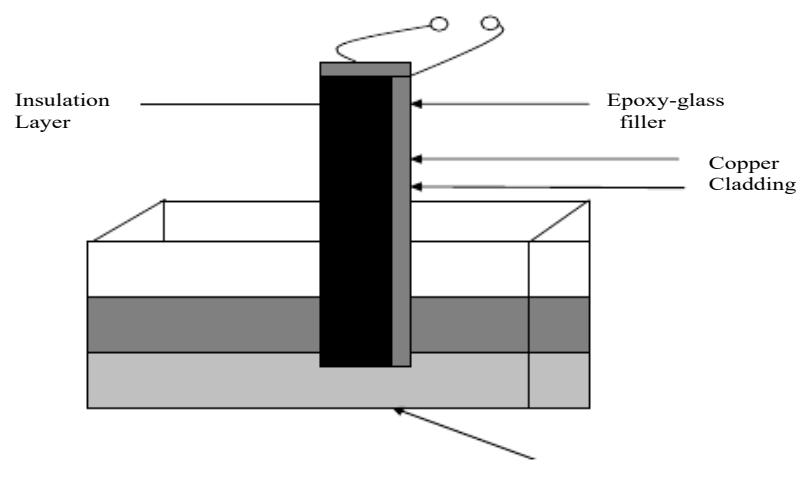

Figure 1 Cross-sectional view of a FOD

In this study, we have made probes with insulation coating of thickness of $5 \mu \mathrm{m}$ to $80 \mu \mathrm{m}$ by preparing $2.5 \%$ to $10 \%$ PMMA solution. The insulation process of the metal electrode of the FODs forms a thin porous film of PMMA on the metal electrode. This porous nature of electrode coated with PMMA can be observed by Scanning Electron Microscopy (SEM) of the electrode surface as shown in fig. 2. The constant phase angle behavior (CPE) of FODs is exhibited due to porosity or roughness of the electrode surface. It is seen from SEM picture that porosity does not appear on the electrode surface which are not coated with PMMA and porosity increases with increase in thickness of coating i.e. pore diameter and number of pores vary with polymer coating (which in tern depends on concentration of PMMA coating). The SEM pictures are shown for various coating thickness. Fig. 2 shows the surface of the 
electrode at the magnification factor 5000 (one with thicker coating and the other with thinner coating).
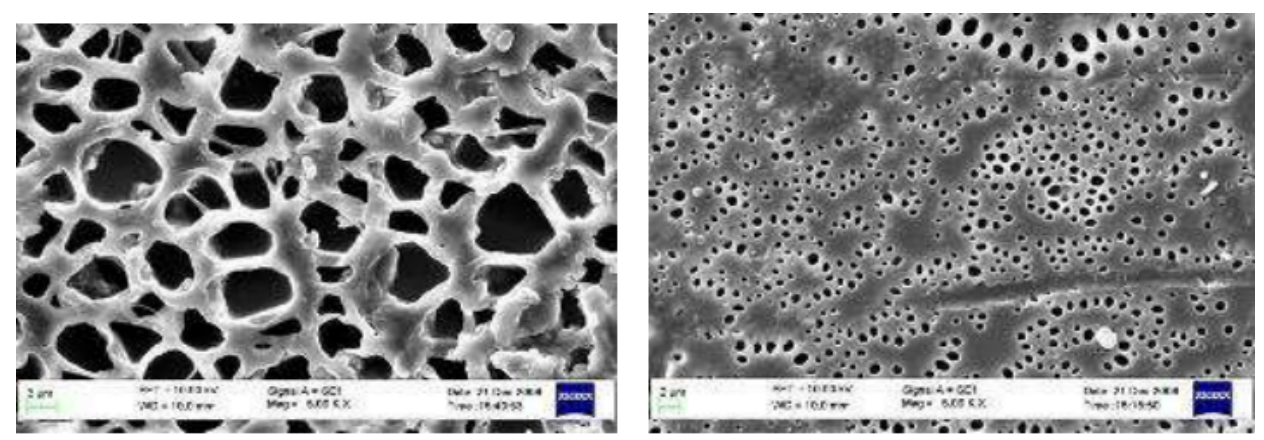

Figure 2 SEM picture of the surface of PMMA coating (a) $85 \mu \mathrm{m}$, coating thickness (b) $15 \mu \mathrm{m}$, coating thickness at a magnification of 5000 .

Final film quality and morphology depends upon the solvent employed, state of substrate surface, deposition rate, type of ionic species and inert additives, all may involved in resulting electrical, mechanical as well as structural feature of polymeric film. The fractional behavior of the FOD is due to the constant phase angle behavior of the device The following section explains the constant phase behavior by measuring the impedance and phase angle of the FODs with a precision LCR meter in $\mathrm{Z}$ and $\theta$ mode

\section{CONSTANT PHASE ANGLE BEHAVIOR OF FRACTIONAL ORDER ELEMENTS (FODs)}

The measurement of impedance and phase angle of the FODs is done with a precision LCR meter in $\mathrm{Z}$ and $\theta$ mode i.e. the equivalent impedance in polar form. An ac excitation of variable frequency and amplitude of $1 \mathrm{~V}$ peak to peak is applied to the electrode. The $\mathrm{Z}$ and $\theta$ of the probe partially dipped in tap water, $\mathrm{pH} 9.2$ solutions, and distilled water, were measured separately at different coating thickness for range of frequency $100 \mathrm{~Hz}$ to $1 \mathrm{MHz}$ as shown in figure.

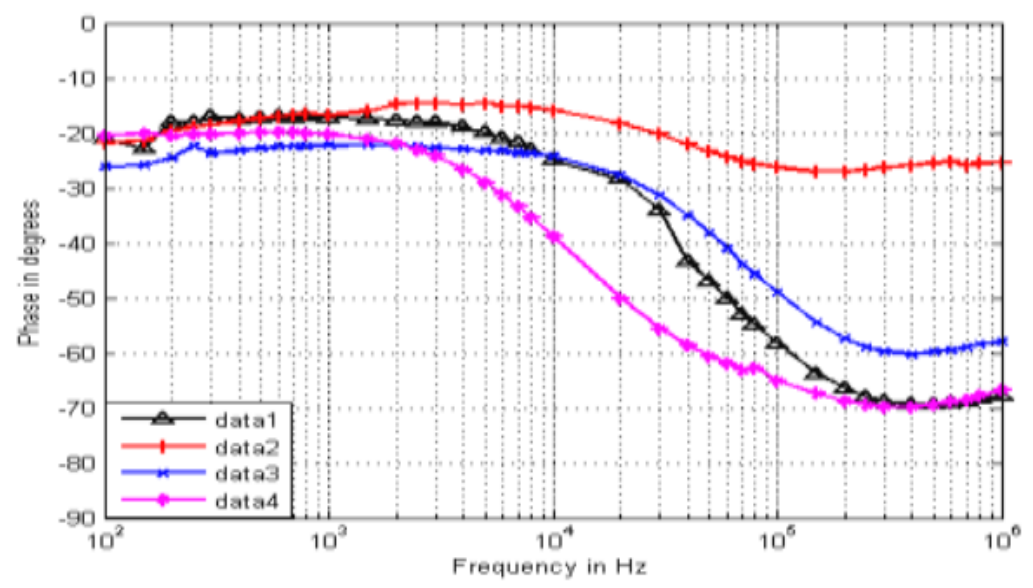

Figure 3(a). Phase plot of FODs 


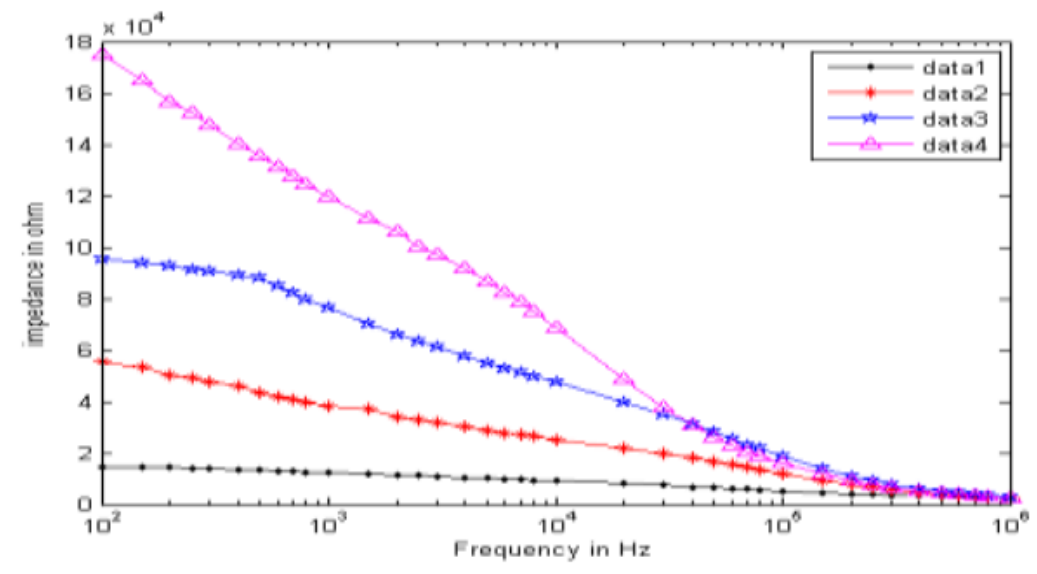

Figure 3 (a). Phase plot of FODs

\section{COMPARISON BETWEEN THE FRACTIONAL ORDER CIRCUITS AND ITS INTEGER ORDER COUNTERPART THROUGH SIMULATION}

\subsection{Exactly fit to the Physical system}

Many physical systems exhibit fractional order behavior. For example: - Infinite transmission line, warburg Impedance, visco-elasticity, flow through weir etc. Hence some design problems may require the circuit characteristics which are not exactly an integer order. So the desired circuit response can not be obtained since available circuit design technique imposes the constrained of an integer-order circuit. For example, based on model parameter of the physical system (which exhibit fractional order behavior), an optimal circuit of order 3.4 is required. This circuit is usually approximated to the nearest realizable order of 4 . However using fractional order circuit, exact order of 3.4 can be obtained by cascading a 2 nd order with 1.4th order circuit.

\subsection{Stop band attenuation any magnitude can be realized.}

Typical stop band attenuation of $-20 \mathrm{~dB} /$ decade for the 2 nd order circuit becomes $-20 \alpha$ $\mathrm{dB} /$ decade for generalized fractional order, where $2 \alpha$ is the order of circuit. Hence, the slope ( $\mathrm{dB} /$ decade) of any magnitude can be realized with fractional order circuit by varying exponent of FOD.

\subsection{Frequency response of fractional order circuits}

The response of the circuits are characterized in terms of circuit parameters (i.e. cut-off frequency, peak frequency, bandwidth etc). This responses of the circuit changes due to the impedance characteristics of the components (capacitors) used in the circuit, which increases or decreases when frequency decreases or increases. Here we have used a FOD in place of a capacitor for realization of the fractional order circuit whose impedance ${ }^{Z=1 / C s^{\alpha}}$. This indicates that the variation of impedance of FOD depends on both frequency and exponent factor $\alpha$. Hence the responses of the fractional order circuit can be varied either by varying exponent / order of the FOD or by varying fractional admittance for a particular frequency. Here the simulation is done for $\mathrm{RC}=0.000515$ for the following transfer functions.

$$
T_{\text {Lowpass }}(s)=\frac{b}{s^{\alpha+\beta}+a s^{\beta}+b}
$$




$$
T_{\text {Highpass }}(s)=\frac{a s^{\alpha+\beta}}{s^{\alpha+\beta}+a s^{\beta}+b}
$$
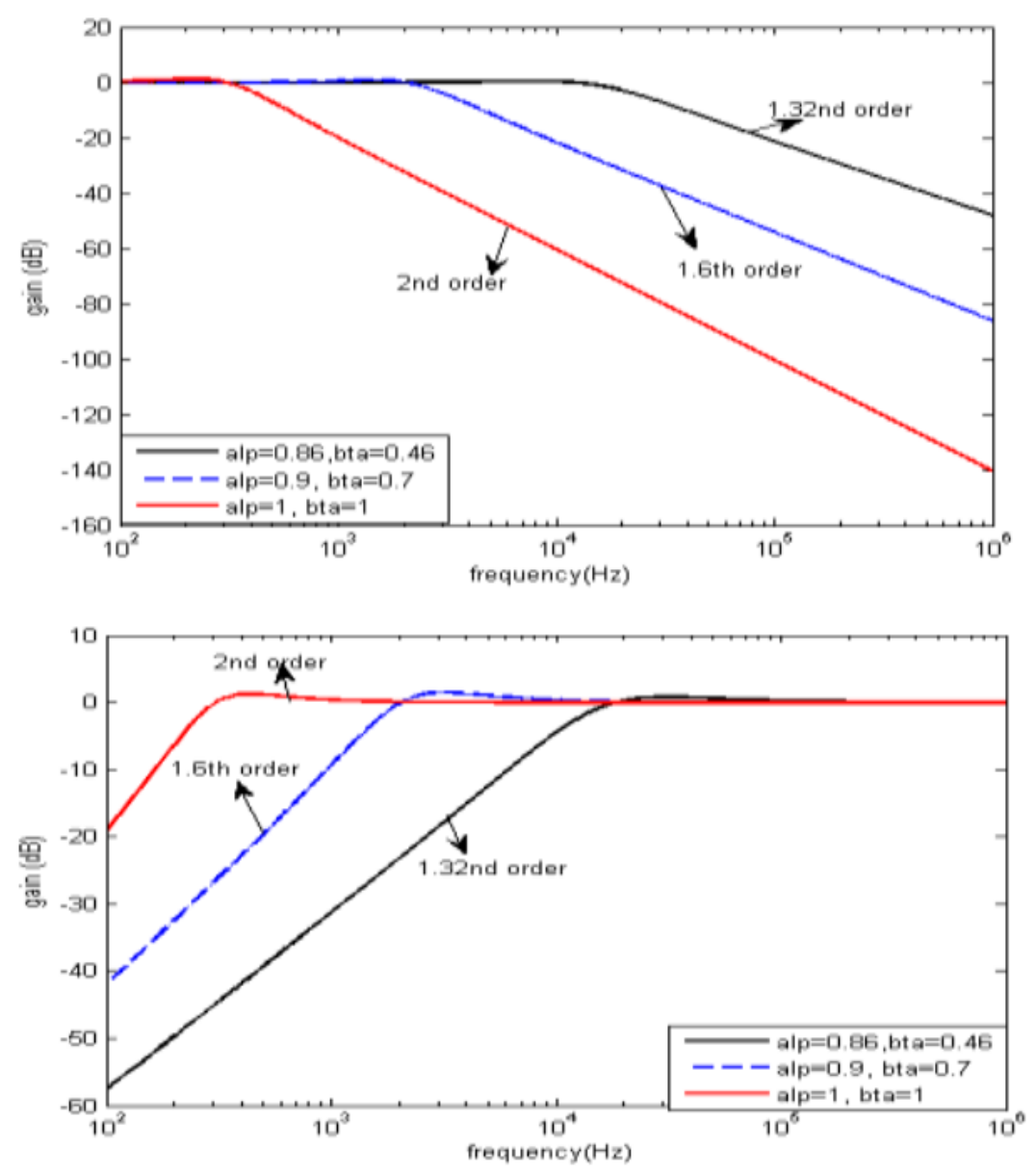

Figure 4 MATLAB simulation of (a) low pass (b) high pass (c) band pass fractional order circuit for different order

\subsection{Improved performance in bandwidth of Fractional order circuit}

The circuit parameters are function of order $(\alpha, \beta)$ of FOD which gives an extra degree of design freedom i.e. we may design circuits for very high frequency application without reducing the RC time constant of the circuit rather changing the exponent factor of the fractional order devices.

For example a cut-off frequency of $0.4 \mathrm{kHz}$ of 2 nd order Low pass circuit can be increased to $21 \mathrm{kHz}$ by changing the exponent factor $(\alpha)$ without reducing the ${ }^{R C}$ time constant as shown in MATLAB simulation (for all cases $R C=0.515 \times 10^{-3}$ ). Similarly the band width of $0.3 \mathrm{kHz}$ of a second order band pass circuit can be increased $24 \mathrm{kHz}$ for a fractional domain. However, slope ( $\mathrm{dB} /$ decade) is decreased by decreasing the order of the circuit, which causes the widening the transition band of the circuit shown in fig. 

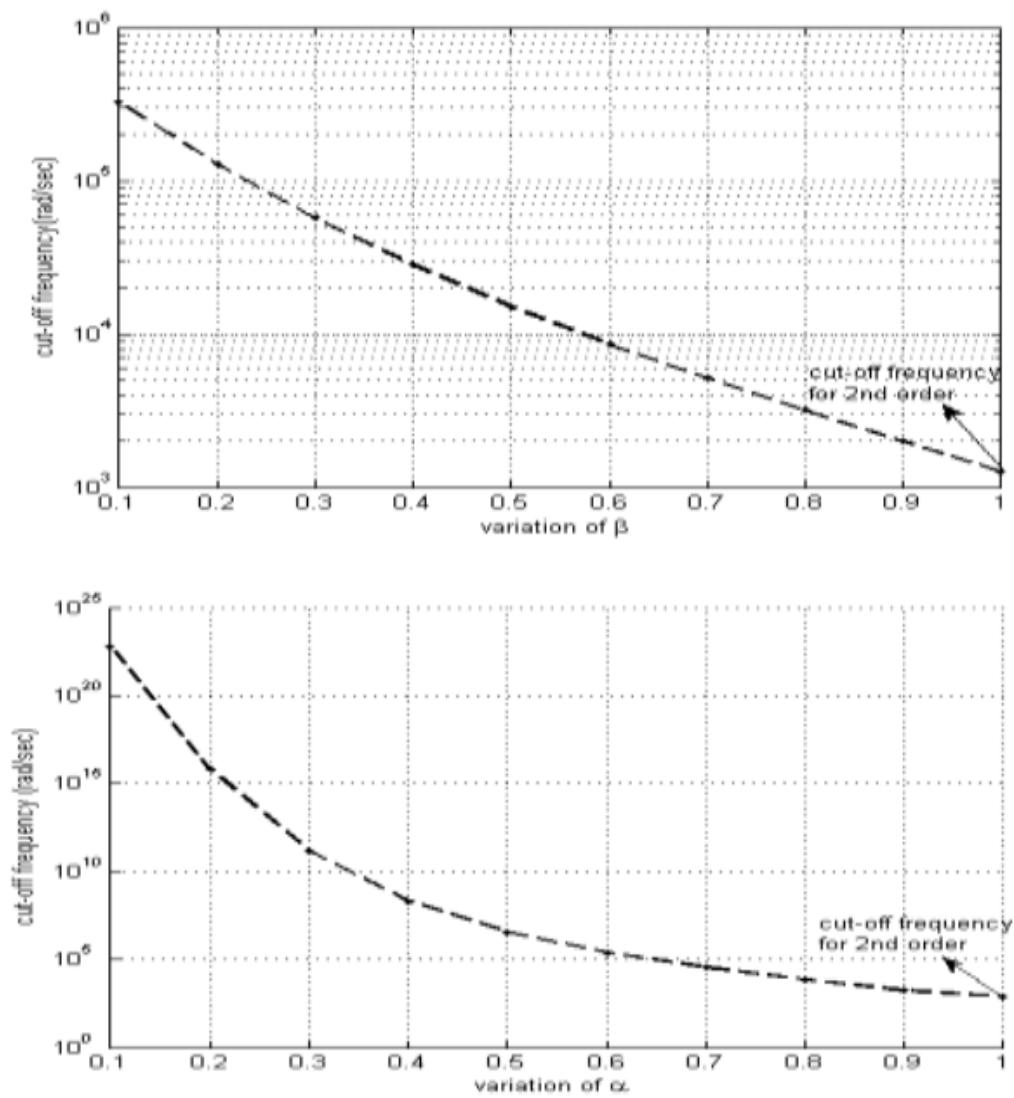

Figure 5 Variation of cut-off frequency for theFOC whose transfer function is described in equation ( 1) and equation (2)

\subsection{Stability in gain of fractional order circuits}

The gain stability range of a fractional order circuit is wider than a conventional integer order circuit.

This can be justified by taking a practical sallen-key (KRC) fractional order low-pass circuit (shown in fig. 6 whose transfer function is given

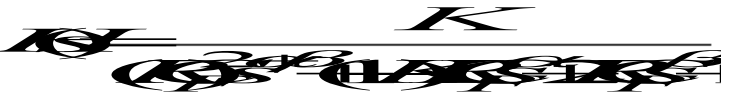

Here $R_{1}=R_{2}=R, C_{F 1}=C_{F 2}=C_{F}$ and $K=1+R_{4} / R_{3}$,

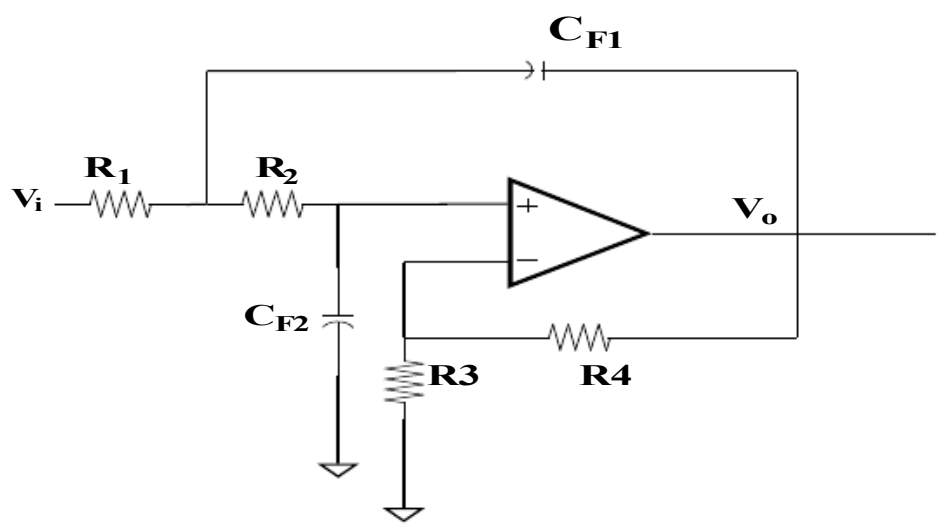

Figure 6 Fractional 2nd order sallen-key filter 
The admittance of two FODs is $Y_{C_{F 1}}=C_{F 1} S^{\alpha}$ and $Y_{C_{F 2}}=C_{F 2} s^{\beta}$ For integer case $(\alpha=\beta=1)$, the filter is unstable when $\mathrm{K}>3$ because the coefficients of the characteristics equation i.e. $\left(R C_{F}\right)^{2} s^{2}+(3-K) R C_{F} s+1=0$ of the 2 nd order filter sallen-key (KRC) become -ve, However, the filter can be stable in fractional domain, when gain $(\mathrm{K})$ is grater than four. The condition of stability [11] for a fractional order filter $(\mathrm{K}>3)$ is given as $n<4 \delta / \pi$, where $\delta=\cos ^{-1} \frac{(K-3) a}{2 \sqrt{b}}<\pi / 2, a^{2} \leq b$ and $\mathrm{n}=$ order of fractional order of filter for the transfer function $T(s)=\frac{b}{s^{2 \alpha}+(3-K) a s^{\alpha}+b}$. So it is clear that the above characteristics equation (with gain $\mathrm{K}=4$ ) can be stable in fractional domain when order is less than 1.33 for $b=a^{2}$.

Hence it is possible to design a stable fractional order sallen-key (KRC) filter for $\mathrm{K}>3$, (i.e. a stable sallen key filter with dc gain greater than 3 can be realized in fractional domain). This can be substantiated by observing the step response of the above filter whose transfer function is given in equation (4) with three conditions i.e.

Case-1: $(\mathrm{k}<3, \alpha=1, \beta=1)$ :-Under such condition, the filter is an integer order (2nd order) sallen-key (KRC) low-pass filter whose dc gain is less than three. From fig. 4(b) it is clear that the step response of the filter converges to a finite steady state value in a finite time and hence stable.

Case-2: $(\mathrm{k}>3, \alpha=1, \beta=1)$ :-Under such condition, the filter is an integer order (2nd order) sallen-key low-pass filter whose dc gain is higher than three (i.e. 4) and become unstable as finite steady state value can't be obtained which can be observed in fig. 4(c).

Case-3 $(\mathrm{k}>3, \alpha=0.8, \beta=0.4)$ :- Under such condition, the filter is a fractional order (1.2nd order) sallen-key (KRC) low-pass filter whose dc gain is higher than three (i.e. 4). Here the step response of the filter converges to steady state value as in case- 1 and hence stable which can be observed in fig. $4(\mathrm{~d})$.

To get the step response of the fractional order sallen-key (KRC) low-pass filter, the fractional order transfer function is approximated to integer order using Oustaloup algorithm in the frequency range $0.1 \mathrm{~Hz}$ to $1 \mathrm{MHz}$ and then MATLAB code discussed in [10] are adopted to plot the step response of above fractional order system.

Here for simulation of both integer and fractional order filter, we have taken $R C_{F}=0.001$

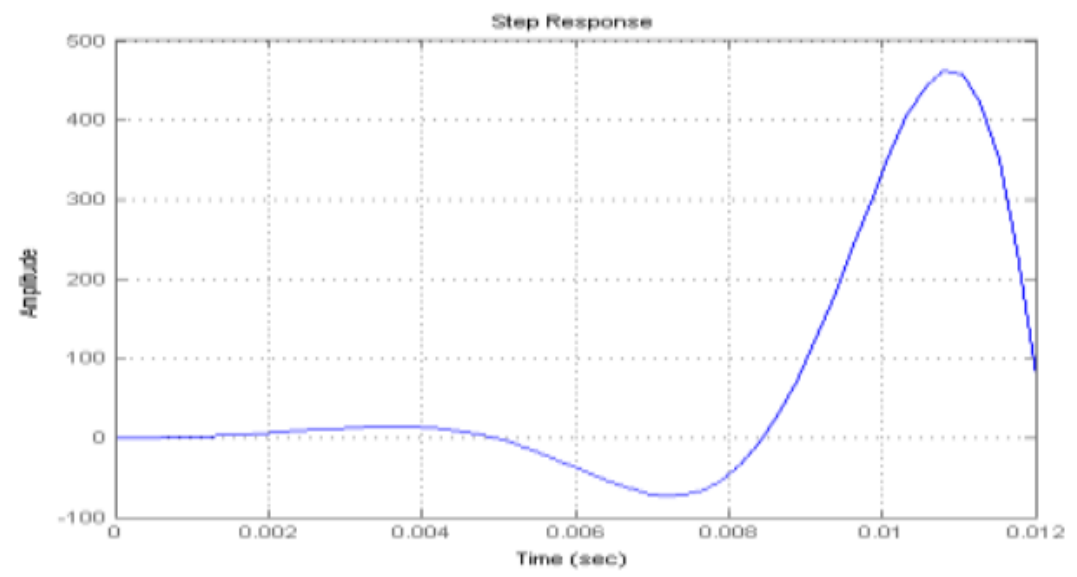

Figure 7 (a) Unit step response of a 2nd order sallen -key low pass filter. (Unstable),

$$
\left(\mathrm{K}=4, \alpha=1, \beta=1, R C_{F}=0.001\right)
$$




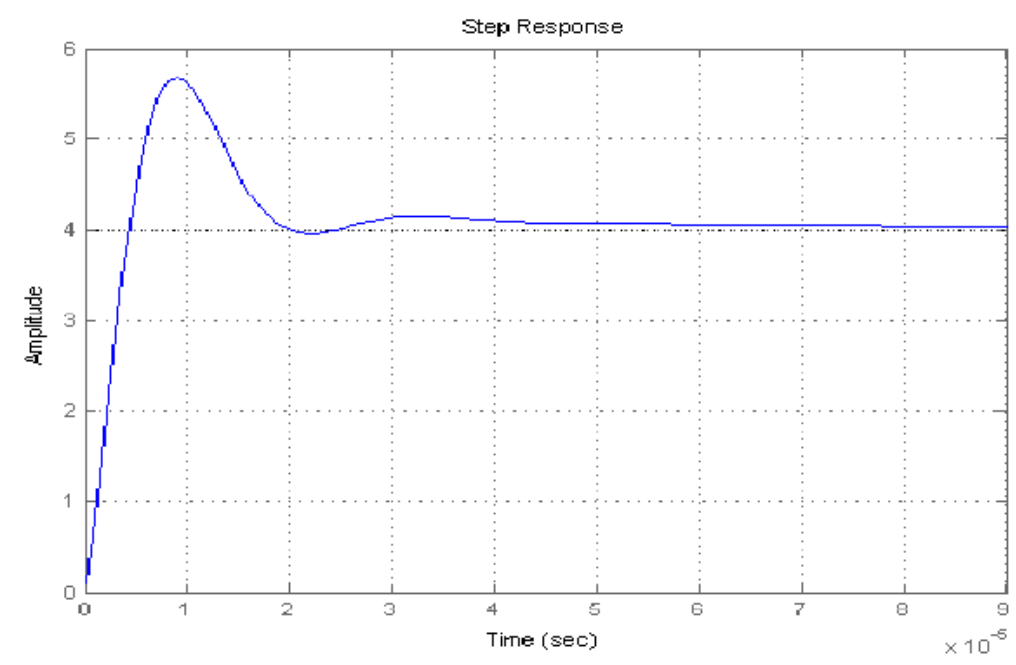

Figure 7 (b) Unit step response of a fractional order sallen -key low pass filter. (Stable)

$$
\left(\mathrm{K}=4, \alpha=0.8, \beta=0.4, R C_{F}=0.001\right.
$$

\section{CONCLUSIONS}

In this work, we have generalized fractional order circuits and their applications. In, particular, fractional order devices are described and realized through simulation work. Also the detailed comparison of fractional order circuits with its integer order counterpart has been presented through simulation. It is noted that FOCs can be used for high frequency application without changing the time constant of the circuits. Similarly the circuit parameters are improved as compared to its integer order counterpart by changing the order of the fractional order device. Being a single component, FODs can be easily used in practical realization of fractional order circuits in futures.

\section{REFERENCES}

[1] K. Biswas, S. Sen and P. Dutta, "Realization of a constant phase element and its performance study in a differentiator circuits”, IEEE Trans.-II, vol. 53, pp. 802-806, 2006.

[2] M.C. Tripathy, D. Mondal, K. Biswas, S. Sen, "Design and performance study of phaselocked loop using fractional-order loop filter", International Journal of Circuit Theory and Applications, 2014.

[3] Madhab Chandra Tripathy,Debasmita Mondal, Karabi Biswas, Siddhartha Sen, "Experimental studies on realization of fractional inductors and fractional order band pass filters", International journal of circuit theory and applications, 2014.

[4] Madhab Chandra Tripathy, Karabi Biswas, Siddhartha Sen, "A design example of a fractional order Kerwin Huelsman- Newcomb biquad filter with two fractional capacitors of different order", Springer science+ business mediaNew York, pp. 1523-1536, 2013.

[5] G. Radwan, A. S. Elwakil and A.M. Soliman, "On the realization of second-order filters to the fractional domain", Journal of circuits and system, vol. 18, No. 2, pp. 361-386, 2009 . 
[6] M. Moshrefi, and J. K. Hammond, "Physical and Geometrical Interpretation of fractional operator", Franklin Inst, vol.335B, No. 6, pp. 1077-1086, 1998.

[7] A.G. Radwan, A. S. Elwakil and A.M. Soliman, "First order filter generalized to the fractional domain", J. circuits syst. Comput. , 17, pp. 55-66, 2008.

[8] G. Carlson and C. halijak, "Approximation of fractional capacitors $\left(\frac{1}{s}\right)^{\frac{1}{n}}$ by regular newton process", IEEE Trans. Circuits System, vol. 11, pp. 210-213, 1964.

[9] I. Petras, "Stability of fractional-order System with rational order", ar Xiv: 0811.4102, vol. 2, [Math. Ds], 2008

[10] Maundy, A. S. Elwakil, T. J. Freeborn, "On the practical realization of higher order filters with a fractional stepping”, signal processing, vol. 91, pp. 484-491, 2011. 